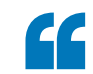

there are no

low-frequency

variants with a

major effect on

DKD risk

$\Rightarrow$ DIABETIC NEPHROPATHY

\title{
The genetic architecture of DKD in T1DM
}

Evidence of a substantial heritability of diabetic kidney disease (DKD) exists but the genetic background is poorly understood. New data confirm that renal complications in type 1 diabetes mellitus (T1DM) are highly heritable and provide insights into the biological mechanisms of DKD.

To comprehensively study the genetic architecture of DKD in T1DM, Niina Sandholm and colleagues - on behalf of the SUMMIT consortium - performed a meta-analysis of genome-wide association studies, which included data from 12,540 individuals. They also used whole-exome sequencing to investigate the effects of low-frequency and rare genetic variants in 997 patients with T1DM.

"We found strong heritability estimates for DKD; additive genetic factors were estimated to explain $35 \%$ of the phenotypic variance and even higher heritability estimates were obtained for more extreme phenotypic definitions," says Sandholm. The analyses did not, however, identify novel susceptibility loci with robust evidence or confirm many previously published associations. The findings suggest that there are no low-frequency variants with a major effect on DKD risk.

Using data from their meta-analysis, the researchers show that sets of alleles that increase body mass index and genetic risk scores for type 2 diabetes are associated with an increased risk of DKD, whereas smoking cessation might have a protective effect. In addition, they identified potential roles of ascorbate and aldarate metabolism and pentose and glucuronate interconversion pathways in the pathogenesis of DKD.

The researchers are now performing a meta-analysis of data from nearly 18,000 patients with T1DM in the JDRF-DNCRI consortium to identify common and lowfrequency genetic variation. They hope that this analysis will identify novel susceptibility loci for DKD and provide biological information in relation to other traits and diseases.

Ellen F. Carney

ORIGINAL ARTICLE Sandholm, N. et al. The genetic

landscape of renal complications in type 1 diabetes. J. Am. Soc. Nephrol. http://dx.doi.org/10.1681/ASN.2016020231 (2016) 